\title{
Association between polymorphisms of the $\alpha$-kinase 1 gene and type 2 diabetes mellitus in community-dwelling individuals
}

\author{
SHIGETAKA SHIMOKATA ${ }^{1}$, MITSUTOSHI OGURI ${ }^{1}$, TETSUO FUJIMAKI ${ }^{2}$, \\ HIDEKI HORIBE $^{3}$, KIMIHIKO KATO ${ }^{4,5}$ and YOSHIJI YAMADA ${ }^{5}$
}

\author{
${ }^{1}$ Department of Cardiology, Japanese Red Cross Nagoya First Hospital, Nagoya, Aichi; ${ }^{2}$ Department of Cardiovascular \\ Medicine, Inabe General Hospital, Inabe, Mie; ${ }^{3}$ Department of Cardiovascular Medicine, Gifu Prefectural \\ Tajimi Hospital, Tajimi, Gifu; ${ }^{4}$ Meitoh Hospital, Nagoya, Aichi; ${ }^{5}$ Department of Human Functional \\ Genomics, Life Science Research Center, Mie University, Tsu, Mie, Japan
}

Received August 05, 2013; Accepted September 13, 2013

DOI: $10.3892 /$ br.2013.173

\begin{abstract}
We previously demonstrated that the $\alpha$-kinase 1 gene $(A L P K 1)$ is a susceptibility locus for chronic kidney disease in individuals with diabetes mellitus (DM) by a genome-wide association study. Although genetic variants of ALPK1 have been associated with chronic kidney disease in individuals with DM, whether $A L P K 1$ is a susceptibility locus for DM has not been elucidated. The purpose of the present study was to investigate a possible association of the rs2074388 $(A \rightarrow G$, Asp565Gly) or rs2074379 $(\mathrm{A} \rightarrow \mathrm{G}$, Ile732Met) variants of $A L P K 1$ with type $2 \mathrm{DM}$ in community-dwelling individuals. The study subjects comprised 5,959 community-dwelling individuals (495 subjects with type $2 \mathrm{DM}$ and 5,464 controls) who were recruited to a population-based cohort study in Inabe, Mie, Japan. The comparisons of allele frequencies or genotype distributions using the Chi-square test revealed that the rs 2074388 and rs2074379 variants of $A L P K 1$ were significantly associated with type $2 \mathrm{DM}(\mathrm{P}<0.05)$. A multivariable logistic regression analysis with adjustment for age, gender, body mass index and smoking status revealed that the $\mathrm{rs} 2074388(\mathrm{P}=0.0051$; odds ratio, 1.32) and rs2074379 ( $\mathrm{P}=0.0058$; odds ratio, 1.32) variants were significantly associated with type $2 \mathrm{DM}$. The haplotype analysis of these polymorphisms revealed that the frequency of the major haplotype, A (rs2074388)-A (rs2074379), was significantly lower, whereas that of the minor haplotype G-G was significantly higher in subjects with type 2 DM compared to controls. Thus, ALPK1 may be a susceptible gene for type $2 \mathrm{DM}$ in community-dwelling Japanese individuals.
\end{abstract}

Correspondence to: Professor Yoshiji Yamada, Department of Human Functional Genomics, Life Science Research Center, Mie University, 1577 Kurima-machiya, Tsu, Mie 514-8507, Japan E-mail: yamada@gene.mie-u.ac.jp

Key words: genetics, polymorphism, type 2 diabetes mellitus, cohort study

\section{Introduction}

The incidence of type 2 diabetes mellitus (DM) is rapidly increasing and has become one of the most common chronic diseases worldwide (1). According to recent estimates, the prevalence of type $2 \mathrm{DM}$ will increase to 439 million adults (7.7\%) by 2030 (2). Asia in particular is a major site of emerging epidemics, mainly due to the transition in nutritional habits and changes in lifestyle (2). Since type $2 \mathrm{DM}$ is associated with an increased incidence of cardiovascular disease and long-term mortality, it imposes a significant economic burden on healthcare wordwide $(3,4)$. Furthermore, the contribution of intensive lifestyle intervention or glycemic control to the reduction of cardiovascular mortality remain controversial $(5,6)$. Therefore, aggressive disease prevention and early detection should be a global strategy priority.

Family history is one of the major risk factors for type 2 DM (7), suggesting that genetic factors significantly contribute to the development and progression of type 2 DM (8). To date, genome-wide association studies (GWASs) have identified various loci and $\sim 40$ genes associated with predisposition to type 2 DM (9-13); however, the genes that contribute to genetic susceptibility to type $2 \mathrm{DM}$ in Japanese individuals have yet to be definitively identified.

We previously demonstrated that the $\alpha$-kinase 1 gene $(A L P K 1)$ is a susceptibility locus for chronic kidney disease in Japanese individuals with DM by a GWAS (14). Although genetic variants of $A L P K 1$ have been associated with chronic kidney disease in individuals with DM, whether $A L P K 1$ is a susceptibility locus for DM has not been elucidated. Thus, we conducted an association study for the rs2074388 $(A \rightarrow G$, Asp565Gly) or rs2074379 (A $\rightarrow \mathrm{G}$, Ile732Met) variants of $A L P K 1$ and type $2 \mathrm{DM}$ in community-dwelling Japanese individuals, in order to provide a basis for the personalized prevention of this disease.

\section{Materials and methods}

Study population. The study population comprised 5,959 community-dwelling individuals (495 subjects with type $2 \mathrm{DM}$ and 5,464 controls) who were recruited to a 
Table I. Characteristics of subjects with type 2 diabetes mellitus (DM) and controls.

\begin{tabular}{|c|c|c|c|}
\hline Characteristics & $\mathrm{DM}$ & Controls & P-value \\
\hline No. of subjects & 495 & 5,464 & \\
\hline Age (years) & $60.2 \pm 9.6$ & $51.9 \pm 12.8$ & $<0.0001$ \\
\hline Gender (male/female, \%) & $74.9 / 25.1$ & $53.6 / 46.4$ & $<0.0001$ \\
\hline Body mass index $\left(\mathrm{kg} / \mathrm{m}^{2}\right)$ & $24.8 \pm 3.8$ & $22.8 \pm 3.3$ & $<0.0001$ \\
\hline Current or former smoker (\%) & 48.7 & 38.5 & $<0.0001$ \\
\hline Hypertension (\%) & 58.2 & 25.3 & $<0.0001$ \\
\hline Systolic blood pressure (mmHg) & $128 \pm 17$ & $119 \pm 16$ & $<0.0001$ \\
\hline Diastolic blood pressure (mmHg) & $78 \pm 12$ & $73 \pm 12$ & $<0.0001$ \\
\hline Dyslipidemia (\%) & 69.3 & 47.0 & $<0.0001$ \\
\hline Serum total cholesterol (mmol/l) & $5.14 \pm 0.94$ & $5.18 \pm 0.84$ & 0.2324 \\
\hline Serum triglycerides $(\mathrm{mmol} / \mathrm{l})$ & $1.52 \pm 1.09$ & $1.23 \pm 0.84$ & $<0.0001$ \\
\hline Serum HDL-cholesterol (mmol/l) & $1.49 \pm 0.39$ & $1.67 \pm 0.44$ & $<0.0001$ \\
\hline Serum LDL-cholesterol (mmol/l) & $3.25 \pm 0.89$ & $3.23 \pm 0.81$ & 0.9922 \\
\hline Chronic kidney disease (\%) & 14.8 & 7.1 & $<0.0001$ \\
\hline Fasting plasma glucose (mmol/l) & $7.85 \pm 2.43$ & $5.34 \pm 0.48$ & $<0.0001$ \\
\hline Blood hemoglobin $\mathrm{A}_{1 \mathrm{c}}(\%)$ & $6.6 \pm 1.3$ & $5.2 \pm 0.3$ & $<0.0001$ \\
\hline Serum creatinine $(\mu \mathrm{mol} / \mathrm{l})$ & $59.6 \pm 14.8$ & $56.0 \pm 13.4$ & $<0.0001$ \\
\hline eGFR (ml min $\left.{ }^{-1} 1.73 \mathrm{~m}^{-2}\right)$ & $77.5 \pm 17.8$ & $80.0 \pm 15.1$ & 0.0002 \\
\hline
\end{tabular}

Quantitative data are presented as means \pm standard deviation. HDL, high-density lipoprotein; LDL, low-density lipoprotein; eGFR, estimated glomerular filtration rate.

population-based cohort study in Inabe, Mie, Japan, between 2010 and 2012. DM was defined as a fasting plasma glucose level of $\geq 6.93 \mathrm{mmol} / 1(126 \mathrm{mg} / \mathrm{dl})$, a blood glycosylated hemoglobin (hemoglobin $\mathrm{A}_{1 \mathrm{c}}$ ) content of $\geq 6.9 \%$, or administration of antidiabetic medication. Type 2 DM was defined according to the criteria of the World Health Organization previously described $(15,16)$. Individuals with type $1 \mathrm{DM}$, maturity-onset diabetes of the young, DM associated with mitochondrial diseases or single gene disorders, pancreatic diseases including severe pancreatitis and pancreatic tumors, other metabolic or endocrinologic diseases, or severe liver and renal dysfunction, were excluded from the study. Individuals on medication that may cause secondary DM were also excluded. The control individuals had a fasting plasma glucose level of $<6.05 \mathrm{mmol} / \mathrm{l}(110 \mathrm{mg} / \mathrm{dl})$ and a blood hemoglobin $\mathrm{A}_{1 \mathrm{c}}$ content of $<6.2 \%$ and had no history of DM or of receiving antidiabetic medication.

The study protocol complied with the Declaration of Helsinki and was approved by the Human Research Ethics Committees of Mie University Graduate School of Medicine and Inabe General Hospital. Written informed consent was obtained from each subject.

Genotyping of polymorphisms. Venous blood $(5 \mathrm{ml})$ was collected into tubes containing $50 \mathrm{mmol} / 1$ ethylenediaminetetraacetic acid (disodium salt), peripheral blood leukocytes were isolated and genomic DNA was extracted from these cells with the SMITEST EX-R\&D DNA extraction kit (Medical and Biological Laboratories, Co., Ltd, Nagoya, Japan). The genotypes of rs2074388 and rs2074379 variants of $A L P K 1$ were determined at G\&G Science Co., Ltd. (Fukushima, Japan) by the multiplex bead-based Luminex assay, a method that combines polymerase chain reaction (PCR) and sequence-specific oligonucleotide probes with suspension array technology (Luminex, Austin, TX, USA). Genotyping involved PCR amplification, hybridization, streptavidin-phycoerythrin reaction and measurement of fluorescence. The genotyping methodology was previously described in detail $(14,17)$.

Statistical analysis. Quantitative data were compared between subjects with type $2 \mathrm{DM}$ and controls using the unpaired Student's t-test and categorical data were compared using the Chi-square test. The allele frequencies were estimated with the gene-counting method and the Chi-square test was used to identify departures from Hardy-Weinberg equilibrium. Genotype distributions and allele frequencies of the rs2074388 and rs2074379 variants of ALPK1 were compared between subjects with type $2 \mathrm{DM}$ and controls using the Chi-square test. A multivariable logistic regression analysis was performed with type $2 \mathrm{DM}$ as a dependent variable and independent variables including age, gender ( 0 , female; 1 , male), body mass index, smoking status $(0$, non-smoker; 1 , current or former smoker) and ALPK1 genotype; the P-values, odds ratios and $95 \%$ confidence intervals were calculated. The ALPK1 genotype was assessed according to dominant (0, wild-type homozygote; 1, heterozygote and variant homozygote) and recessive (0, wild-type homozygote and heterozygote; 1 , variant homozygote) genetic models. We investigated the linkage disequilibrium between the rs2074388 and rs2074379 variants of $A L P K 1$ and the association of the haplotypes of these polymorphisms to type $2 \mathrm{DM}$. $\mathrm{P}<0.05$ was considered to indicate statistically significant differences. Statistical significance was assessed using two-sided tests performed with JMP 
Table II. Comparison of genotype distributions or allele frequencies of the rs 2074388 and rs 2074379 variants of $A L P K 1$ by the Chi-square test between subjects with type 2 diabetes mellitus (DM) and controls.

\begin{tabular}{lccc}
\hline Genotypes & DM $^{\mathrm{a}}$ & Controls $^{\mathrm{a}}$ & P-value (genotype) \\
\hline rs2074388 & & & 0.0244 \\
AA & $197(39.8)$ & $2517(46.1)$ & \\
AG & $239(48.3)$ & $2393(43.8)$ & \\
GG & $59(11.9)$ & $554(10.1)$ & \\
Hardy-Weinberg P-value & 0.2953 & 0.6723 & 0.0304 \\
rs2074379 & & & \\
AA & $198(40.0)$ & $2516(46.0)$ & \\
AG & $238(48.1)$ & $2397(43.9)$ & \\
GG & $59(11.9)$ & $551(10.1)$ & \\
Hardy-Weinberg P-value & 0.3283 & 0.5689 & \\
\hline
\end{tabular}

${ }^{a}$ The numbers in parentheses are percentages. $A L P K 1, \alpha$-kinase 1 gene.

Table III. Multivariable logistic regression analysis of the rs2074388 and rs2074379 variants of ALPK1 and type 2 diabetes mellitus with adjustment for age, gender, body mass index and smoking status.

\begin{tabular}{llccc}
\hline & \multicolumn{2}{c}{ Dominant } & & \multicolumn{2}{c}{ Recessive } \\
\cline { 2 - 4 } Genotypes & P-value & OR $(95 \% \mathrm{CI})$ & P-value & OR (95\% CI) \\
\hline rs2074388 $(\mathrm{A} \rightarrow \mathrm{G})$ & 0.0051 & $1.32(1.09-1.61)$ & 0.1693 & - \\
rs2074379 $(\mathrm{A} \rightarrow \mathrm{G})$ & 0.0058 & $1.32(1.08-1.60)$ & 0.1259 & - \\
\hline
\end{tabular}

OR, odds ratio; CI, confidence interval; $A L P K 1$, $\alpha$-kinase 1 gene.

Table IV. Association of $A L P K 1$ haplotypes with type 2 diabetes mellitus (DM).

\begin{tabular}{|c|c|c|c|c|c|}
\hline \multirow[b]{2}{*}{ Haplotype } & \multirow[b]{2}{*}{ Overall frequency } & \multicolumn{2}{|c|}{ Frequency } & \multirow{2}{*}{$\begin{array}{c}\text { Chi-square } \\
\text { P-value }\end{array}$} & \multirow{2}{*}{$\begin{array}{c}\text { Permutation } \\
\text { P-value }\end{array}$} \\
\hline & & $\mathrm{DM}$ & Controls & & \\
\hline A-A & 0.6754 & 0.6394 & 0.6787 & 0.0115 & 0.015 \\
\hline $\mathrm{G}-\mathrm{G}$ & 0.3226 & 0.3596 & 0.3193 & 0.0094 & 0.010 \\
\hline G-A & 0.0011 & 0.0010 & 0.0011 & 0.9353 & 0.652 \\
\hline$A-G$ & 0.0008 & $1.6 \times 10^{-12}$ & 0.0009 & 0.3407 & 0.358 \\
\hline
\end{tabular}

Haplotypes consist of the $\mathrm{A} \rightarrow \mathrm{G}(\mathrm{rs} 2074388)$ and $\mathrm{A} \rightarrow \mathrm{G}(\mathrm{rs} 2074379)$ of $A L P K 1$. ALPK1, $\alpha$-kinase 1 gene.

Genomics software, version 6.0 (SAS Institute, Cary, NC, USA). Linkage disequilibrium and haplotype analysis of these polymorphisms were performed with SNPAlyze software, version 6 (Dynacom Co., Ltd., Yokohama, Japan).

\section{Results}

Subject characteristics. The baseline characteristics of the subjects are presented in Table I. Age, the frequency of male gender, body mass index and the prevalence of smoking, hypertension, dyslipidemia and chronic kidney disease were higher among subjects with type 2 DM compared to controls.
Comparison of genotype distributions and allele frequencies. The comparison of genotype distributions or allele frequencies using the Chi-square test revealed that the rs2074388 and rs2074379 variants of $A L P K 1$ were significantly associated with type $2 \mathrm{DM}(\mathrm{P}<0.05)$ (Table II). The genotype distributions of the two polymorphisms were in Hardy-Weinberg equilibrium among subjects with type $2 \mathrm{DM}$ and controls.

Multivariable logistic regression analysis. A multivariable logistic regression analysis with adjustment for age, gender, body mass index and smoking status revealed that rs2074388 (dominant model) and rs2074379 (dominant model) were 
significantly associated with type 2 DM, with the $G$ alleles of the two polymorphisms representing risk factors for this disease (Table III).

Haplotype analysis. As the rs2074388 and rs2074379 variants of $A L P K 1$ were in linkage disequilibrium [standard linkage disequilibrium coefficient $\left.\left(\mathrm{r}^{2}\right)=0.9908, \mathrm{P}<0.0001\right]$, we performed a haplotype analysis for these polymorphisms. That analysis revealed that the frequency of the major haplotype, A (rs2074388)-A (rs2074379), was significantly lower $(\mathrm{P}<0.05)$, whereas that of the minor haplotype $\mathrm{G}-\mathrm{G}$ was significantly higher in subjects with type 2 DM compared to controls (Table IV).

Association of ALPK1 polymorphisms with plasma glucose and hemoglobin $A_{l c}$. Finally, we assessed the association of rs2074388 or rs2074379 to fasting plasma glucose level or blood hemoglobin $\mathrm{A}_{1 \mathrm{c}}$ content among all individuals, or individuals not on antidiabetic medication. There were no significant differences in the fasting plasma glucose level or blood hemoglobin $\mathrm{A}_{1 \mathrm{c}}$ content among the of rs2074388 or rs2074379 genotypes (data not shown).

\section{Discussion}

We previously demonstrated that $A L P K 1$ is a susceptibility locus for chronic kidney disease in individuals with DM by a GWAS (14). In this study, we demonstrated that the rs2074388 and rs2074379 variants of ALPK1 were significantly associated with the prevalence of type $2 \mathrm{DM}$ in community-dwelling Japanese individuals, with the $G$ alleles of the two polymorphisms representing risk factors for this disease.

ALPK1 is a member of a newly discovered protein kinase family that exhibits no sequence homology to the conventional protein kinases (18). It functions in apical transport by phosphorylating myosin-1a and is a putative candidate for the regulation of intracellular trafficking processes by phosphorylation (19). ALPK1 may act synergistically with monosodium urate monohydrate crystals in promoting the production of proinflammatory cytokines through the activation of nuclear factor- $\kappa \mathrm{B}$ and mitogen-activated protein kinase (ERK1/2 and p38) signaling in cultured HEK293 cells, suggesting that ALPK1 may contribute to the inflammatory process associated with the development of gout (20).

Impaired insulin secretion and increased insulin resistance are key components of type 2 DM (21). Although the contributions of these factors to the onset and progression of type 2 DM may differ between Caucasian and Asian populations, both factors are essential for the diagnostic and therapeutic strategies of type 2 DM (22). Recent studies demonstrated that proinflammatory cytokines (interleukin- $1 \beta$ and tumor necrosis factor) exert deleterious effects on insulin secretion and insulin resistance $(23,24)$. In addition, signal pathways activated by proinflammatory cytokines, such as nuclear factor- $\kappa \mathrm{B}$ signaling, were shown to be responsible for these conditions (25). As chronic inflammation may be an important factor in the development of type $2 \mathrm{DM}$, the effects of the rs2074388 or rs2074379 variants of ALPK1 on the acceleration of the inflammatory process may account for their association with type $2 \mathrm{DM}$.
Although the rs2074388 and rs2074379 variants of $A L P K 1$ were significantly associated with the prevalence of type 2 DM, either polymorphism was not associated with fasting plasma glucose level or blood hemoglobin $\mathrm{A}_{1 \mathrm{c}}$ content among all individuals or individuals not on antidiabetic medication. Although the reason for this discrepancy has not been fully elucidated, there are possible explanations: i) the number of subjects with type 2 DM not on antidiabetic medication was limited; ii) information regarding drug compliance obtained by a questionnaire was incomplete; and iii) either polymorphism was not associated with fasting plasma glucose level among control individuals.

Our study had certain limitations: i) as the results of the present study were not replicated, validation of our findings may require their replication with other independent subject panels or ethnic groups; ii) it is possible that rs2074388 or rs2074379 are in linkage disequilibrium with other polymorphisms in the same gene or in other nearby genes that are actually responsible for the development of type 2 DM; iii) although the rs2074388 and rs2074379 variants of $A L P K 1$ were significantly associated with the prevalence of type $2 \mathrm{DM}$, either polymorphism was not associated with fasting plasma glucose level or blood hemoglobin $\mathrm{A}_{1 \mathrm{c}}$ content; and iv) the functional relevance of the rs2074388 or rs2074379 variants of $A L P K 1$ to the pathogenesis of type $2 \mathrm{DM}$ has not been determined.

In conclusion, our results suggest that $A L P K 1$ is a susceptibility gene for type $2 \mathrm{DM}$ in community-dwelling Japanese individuals. Determination of the genotypes for the polymorphisms of ALPK1 may prove informative for the assessment of the genetic risk for type $2 \mathrm{DM}$ in the Japanese population.

\section{Acknowledgements}

This study was supported by a Grant-in-Aid for Scientific Research from the Ministry of Education, Culture, Sports, Science and Technology of Japan (no. 24590746 to Y.Y.) and by Research Grants from the Japan Health Foundation and Okasan Kato Culture Promotion Foundation (to Y.Y.).

\section{References}

1. Shaw JE, Sicree RA and Zimmet PZ: Global estimates of the prevalence of diabetes for 2010 and 2030. Diabetes Res Clin Pract 87: 4-14, 2010.

2. Chan JC, Malik V, Jia W, et al: Diabetes in Asia: epidemiology, risk factors, and pathophysiology. JAMA 301: 2129-2140, 2009.

3. American Diabetes Association: Economic costs of diabetes in the U.S. in 2012. Diabetes Care 36: 1033-1046, 2013.

4. Zhang P, Zhang X, Brown J, et al: Global healthcare expenditure on diabetes for 2010 and 2030. Diabetes Res Clin Pract 87: 293-301, 2010.

5. Dailey G: Early and intensive therapy for management of hyperglycemia and cardiovascular risk factors in patients with type 2 diabetes. Clin Ther 33: 665-678, 2011.

6. Look AHEAD Research Group; Wing RR, Bolin P, Brancati FL, et al: Cardiovascular effects of intensive lifestyle intervention in type 2 diabetes. N Engl J Med 369: 145-154, 2013.

7. Valdez R: Detecting undiagnosed type 2 diabetes: family history as a risk factor and screening tool. J Diabetes Sci Technol 3: 722-726, 2009.

8. Hu FB: Globalization of diabetes: the role of diet, lifestyle, and genes. Diabetes Care 34: 1249-1257, 2011.

9. Yamauchi T, Hara K, Maeda S, et al: A genome-wide association study in the Japanese population identifies susceptibility loci for type 2 diabetes at UBE2E2 and C2CD4A-C2CD4B. Nat Genet 42: 864-868, 2010. 
10. Tabassum R, Chauhan G, Dwivedi OP, et al: Genome-wide association study for type 2 diabetes in Indians identifies a new susceptibility locus at 2q21. Diabetes 62: 977-986, 2013.

11. Cho YS, Chen $\mathrm{CH}, \mathrm{Hu} \mathrm{C}$, et al: Meta-analysis of genome-wide association studies identifies eight new loci for type 2 diabetes in east Asians. Nat Genet 44: 67-72, 2011.

12. Kooner JS, Saleheen D, Sim X, et al: Genome-wide association study in individuals of South Asian ancestry identifies six new type 2 diabetes susceptibility loci. Nat Genet 43: 984-989, 2011.

13. Sladek R, Rocheleau G, Rung J, et al: A genome-wide association study identifies novel risk loci for type 2 diabetes. Nature 445: 881-885, 2007.

14. Yamada Y, Nishida T, Ichihara S, et al: Identification of chromosome 3q28 and ALPK1 as susceptibility loci for chronic kidney disease in Japanese individuals by a genome-wide association study. J Med Genet 50: 410-418, 2013

15. American Diabetes Association: Report of the expert committee on the diagnosis and classification of diabetes mellitus. Expert Committee on the Diagnosis and Classification of Diabetes Mellitus: Diab Care 26 (Suppl): S5-S20, 2003.

16. Kuzuya T, Nakagawa S, Satoh J, et al; Committee of the Japan Diabetes Society on the diagnostic criteria of diabetes mellitus: Report of the Committee on the classification and diagnosis criteria of diabetes mellitus. Diabetes Res Clin Pract 55: 65-85, 2002.

17. Itoh Y, Mizuki N, Shimada T, et al: High-throughput DNA typing of HLA-A, -B, -C, and -DRB1 loci by a PCR-SSOP-Luminex method in the Japanese population. Immunogenetics 57: 717-729, 2005 .
18. Ryazanov AG, Pavur KS and Dorovkov MV: Alpha-kinases: a new class of protein kinases with a novel catalytic domain. Curr Biol 9: R43-R45, 1999.

19. Heine M, Cramm-Behrens CI, Ansari A, et al: Alpha-kinase 1, a new component in apical protein transport. J Biol Chem 280: 25637-25643, 2005.

20. Wang SJ, Tu HP, Ko AM, et al: Lymphocyte $\alpha$-kinase is a gout-susceptible gene involved in monosodium urate monohydrate-induced inflammatory responses. J Mol Med 89: 1241-1251, 2011.

21. Kahn SE: The relative contributions of insulin resistance and beta-cell dysfunction to the pathophysiology of type 2 diabetes. Diabetologia 46: 3-19, 2003.

22. Fukushima M, Usami M, Ikeda M, et al: Insulin secretion and insulin sensitivity at different stages of glucose tolerance: a cross-sectional study of Japanese type 2 diabetes. Metabolism 53: 831-835, 2004.

23. Ruotsalainen E, Salmenniemi U, Vauhkonen I, et al: Changes in inflammatory cytokines are related to impaired glucose tolerance in offspring of type 2 diabetic subjects. Diab Care 29: 2714-2720, 2006.

24. Olefsky JM and Glass CK: Macrophages, inflammation, and insulin resistance. Annu Rev Physiol 72: 219-246, 2010.

25. Norlin S, Ahlgren U and Edlund $\mathrm{H}$ : Nuclear factor- $\kappa \mathrm{B}$ activity in $\beta$-cells is required for glucose-stimulated insulin secretion. Diabetes 54: 125-132, 2005 . 